\title{
A Monitoring and Management System for Farmland Environmental Base on Flex and Web Services"
}

\author{
Yanmin Wang ${ }^{1,2}$, Yuchun Pan ${ }^{2, * *}$, Bingbo $\mathrm{Gao}^{2}$, Zhenyu Zhang ${ }^{1,3}$, and Bingjun $\mathrm{Li}^{1}$ \\ ${ }^{1}$ College of Information and Management Science, Henan Agricultural University, \\ 450002, Henan, P.R. China \\ ${ }^{2}$ Beijing Research Center for Information Technology in Agriculture, \\ 100097, Beijing, P.R. China \\ ${ }^{3}$ Department of Industry and Information Technology of Henan Province, \\ 450008, Henan, P.R. China \\ wy_minasina.cn, \\ panyc@nercita.org.cn
}

\begin{abstract}
Farmland environmental deterioration is the main reason which results in the agricultural product quality problems, so it is necessary to build a geospatial information system for farmland environmental monitoring and management. The rapid development of portable farmland environmental surveying or sampling devices and sensor network have made this possible. In this paper, we describe the design and implement of the geospatial system based on Flex and Web Services. This system provides data management, geoprocessing, visualization, as well as analysis functions to mine potential valuable information to support decision making based on the sampling data and other collected environmental data of farmland. Geoprocessing is implemented by directly invoking Geo-processing Web Services or calling Server APIs. To enhance the usability, the system also provides a flex client for users and web service interfaces for client program. Tertiary user structure with strict privileges management is designed to protect the users' data copyright.
\end{abstract}

Keywords: Geospatial Information System, Farmland Environmental Management and Monitoring, Geospatial Web Services, Flex.

\section{Introduction}

The security of agricultural food has been one of the most concerned issues in most countries in the world. The agricultural product quality problem has mainly come from farmland environmental deterioration. The overuse of fertilizers, abuse of pesticides and the heavy metal pollution are the main causes of the environmental deterioration of farmlands. In China, 1.6 million hectares of farmland has been polluted by pesticides, 4.8 million tons of fertilizers are being applied to the farmland

\footnotetext{
Beijing Municipal Natural Science Foundation (4102022)\& key Projects for Constructing Scientific and Technological Innovation Ability of Beijing Academy of Agriculture and Forestry Sciences.

** Corresponding author.
} 
every year[1], and more than 20 million hectares of farmland (that's more the one fifth of the total) have been contaminated by heavy metal [2-4]. The Chinese government is paying more and more attentions on the farm land environmental quality and has initiated a series of projects to control the contamination and repair the contaminated soil. To achieve these goals, the primary thing needed to perform is to fig out the pollution status of farmland. The development of sensor network makes it possible to monitor and the emergence of portable sampling equipments with acceptable prices such as TXRF7 and X-MET5000 makes it possible to build farmland environmental information base [5-6]. However, the management and utilizing ability of the collected farmland environment data is very limited in China.

As an effective technology to manage, display, process and analyze the spatial related data, GIS (Geographical Information System) has been applied into many fields. By using WebGIS technology the spatial information and GIS application can be shared over the Internet easily. The ESRI ArcGIS Server provides a flexible framework to create and configure GIS services and applications [7-8], while Adobe Flex which has distinct advantage over traditional web technologies in giving an expressive interface and more effective communication mechanism becomes the most popular RIA (Rich Internet Application) technology. Therefore, in this paper, Web Services, ArcGIS Server, Flex as well as J2EE technology are employed to build the MMSFEFW(abbreviation of A Management and Monitoring System for Farmland Environmental base on Flex and Web Services) to effectively manage, display and analyze the collected farmland environmental data. The design, key technologies and implementation of MMSFEFW are discussed in section 2, section 3 and section 4, respectively.

\section{System Design}

\subsection{System Structure}

The MMSFEFW is composed of data layer, service layer and view layer, shown in fig 1.The data layer contains base data, sampling data, information of system users which can be spatial or non-spatial data. The spatial data is managed by relational DBMS (database management system) and the spatial data engine (in this paper the ArcSDE of ESRI is adopted). The non-spatial data are stored in the relational database and managed by relational RDBMS (Relational Database Management System).

The service layer is composed of spatial service layer, application logic layer and the interaction layer. The ArcGIS Server is paced in the spatial service layer to provide spatial information and functions through spatial services and Server API. The application logic layer acts as the Model of MVC (Model-View-Controller) architecture. It contains the basic function module which encapsulates the common supporting functions into Classes of appropriate granularity and the logic module carrying out the system functions by calling the basic function module. The interaction layer, working between clients and the application logic layer, contains web module for web browsers and web service for client program. The former plays 
as the controller of MVC architecture and the latter provides standard interface for client program to access system functions.

The view layer is designed to possess two kinds of interface, they are flex interface for users and Web Services interface such as WSDL (Web Service Description Language) for client program. The flex client, written using action script and Adobe tags would finally be compiled to a swf (shock wave flash, a binary file of Adobe Company) files and downloaded to users' web browsers to provide a vivid interface which communicates with the server to get data, write data and call functions according to users' operations. The Web Services interface provides service invoking information such as address, operations, input and output parameters and accepts users' request in SOAP (Simple Object Access Protocol) Envelope.

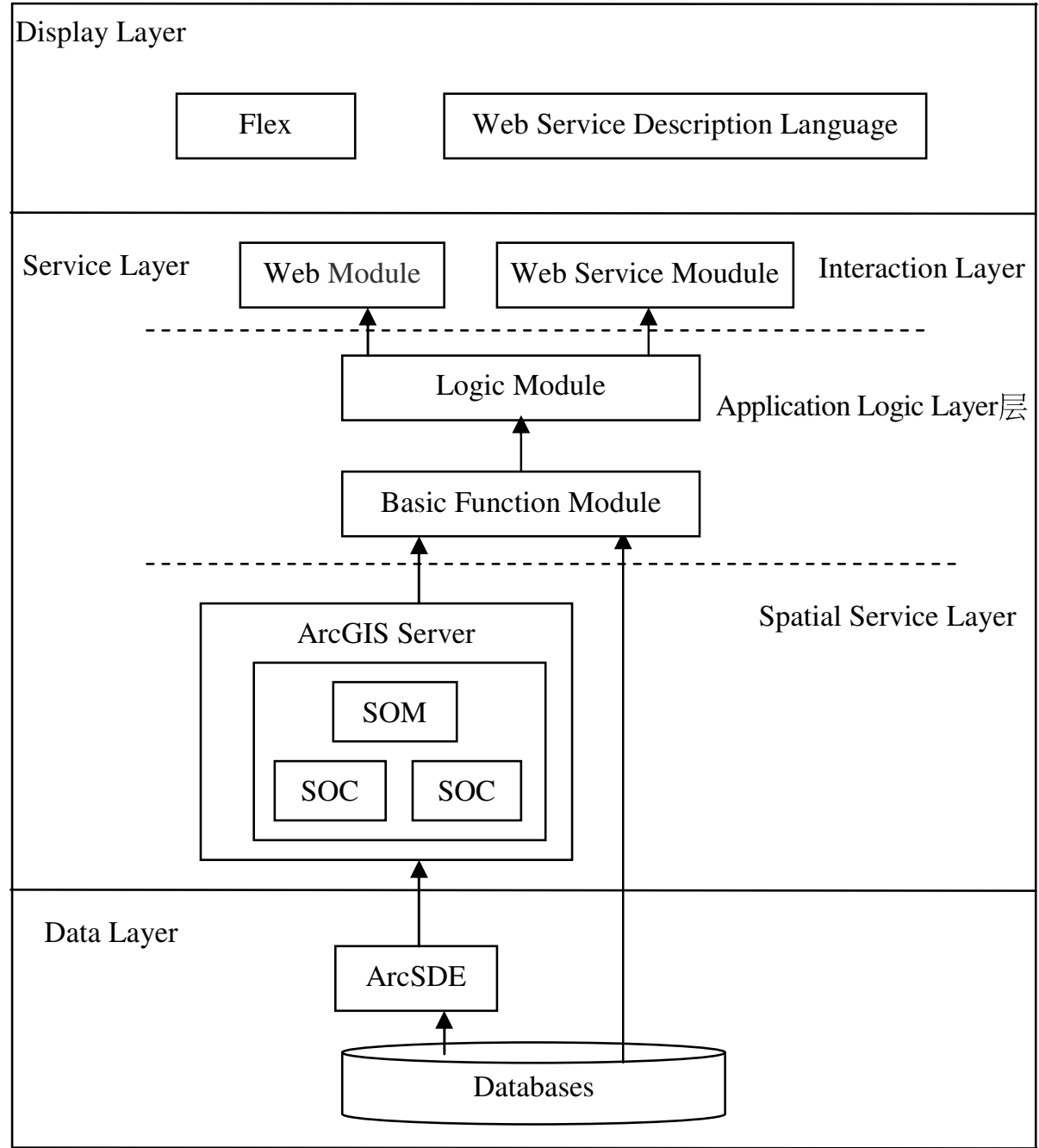

Fig. 1. System structure 


\subsection{System Functions}

Aimed at managing, visualizing and analyzing the detection data of environment sampling data of farmland, mining the potential value of the collected data, serving data collector, data manager, experts and researchers, related government officials and the public, the MMSFEFW is designed to contain three function groups, namely data management group, query and analysis group, and system management group, shown in fig 2.

(1) Data management group: This group contains functions that support the upload, query and editing of the farmland plot data and the sampling data collected by fixed monitoring sites or survey activity. For the plots data many common GIS data formats are supported, such as Shape file of ESRI, GML and so on. The sampling data can be uploaded in Microsoft Excel file, plain text file or XML file.

(2) Query and analysis group: this group includes functions to query data using attributes restrictions and spatial location or relationship restrictions, apply spatial analysis on the sampling data, evaluate the quality farmland plots, display the results of analysis and evaluations on thematic maps and support users to set levels and rendering colors the results.

(3) System management group: the system configuration function and user management function are in this group. System configuration function supports system administrators to set system parameters and user management function provides medium to manager users, user groups and their privileges.



Fig. 2. Functional block diagram of system 


\subsection{Data Organization}

The data of MMSFEFW include basic data, sampling data of farmland environment and user information. Basic data are composed of different kinds of data, including remote sensing images, terrain data, administrative map, and land use data and so on. The sampling data include observation data of soil, atmosphere environment and water environment of farmland and the metadata describing the observation data. User information includes basic user information and the role and privileges assigned to the user.

Google map is employed to provide background map so images and terrain maps were not built into the database. Other basic data like administrative map, land use map, were publish into ArcGIS Server to provide web map service after being organized into different map documents and given proper styles and visible ranges. Because those background data are stable and do not change frequently, map tiles are created beforehand at 20 scale levels same as Google map. After that, when the server received a web map request, it only needs to return the tiles within the extent at proper scale level. Thus the responsive time is shorted sharply for there is no need to render the map in-time.

The sampling data were represented by point feature type with a point geometry attribute and other non-spatial sampling attributes. The farmland plots data were represented by polygon feature types with a point geometry attribute and other nonspatial attributes. Because the number of non-spatial attributes of sampling data and farmland plots can be accustomed and thus is not fixed, the spatial attributes and the non-spatial attributes are stored in different tables. For example the sampling data, the points was stored in a spatial data table and other attributes are stored in normal table and they are connected with the object id. The metadata of the non-spatial attributes are stored in the meta-table table and the M_Sample_Fields table. Every sampling point and farmland plots has an attributes named "owner" attribute showing which user it belongs to. Besides the spatial index built on the geometry attributes, index must be created on the "owner" attribute because the data access control which is very frequent in MMSFEFW is base on it.

Three tier user structure (induced in 4.3) is adopted in MMSFEFW, so user information, user group information, roles and privileges need to be stored. User table, group table, roles table and privileges table were created to store this information.

\section{Key Technologies}

\subsection{ArcGIS Server}

ArcGIS Server provides the framework to create and configure GIS services and applications. It is a GIS web server software supporting users to configure and publish spatial resources (data, map, functions) as REST style Web Services or SOAP style Web Services, for example mapping service, image service, spatial data service, geocoding service and spatial processing service. Two steps are required to publish a spatial resource as web service: first create a spatial resource use the ArcGIS Desktop, then publish the resource into ArcGIS Server through ArcCatalog or the ArcGIS Server Manager. 
ArcGIS Server provides developers with Server APIs which are actually coarsegrained ArcObjects to connect and get objects from the server. Developers can invoke fine-grained ArcObjects through the Server APIs to fully employ the powerful spatial data process and analysis ability of ArcGIS. The architecture of calling ArcObjects of ArcGIS Server in Web Application is shown in Fig 3. The web application server acts as a bridge that connects web clients to the spatial operation and analyzing functions of ArcGIS Server. The web application server and GIS server can reside in different computers. The web application written in java or .net uses ArcObjects proxies which then communicate with the SOM (Server Object Manager) using XML format messages and HTTP protocol to revoke the ArcObjects in the SOC (Server Object Containers). What need to note is that it's the ArcObjects residing in the SOC that actually implement the spatial operation or analysis functions.

ArcGIS Server also offers developers ADF (Application Developer Framework), a robust, standards-based set of components for building and deploying geospatial applications and services. For Java platform, there are the Web ADF for building and deploying Web applications and services base on JSF (Java Server Faces) and Enterprise ADF components for deploying business-tier EJB (Enterprise JavaBeans) applications [9-12].

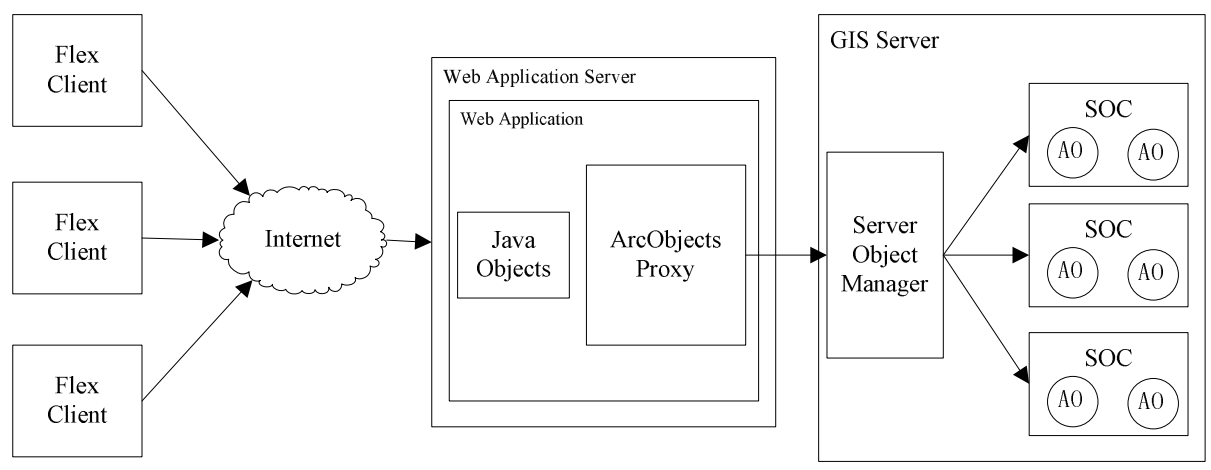

Fig. 3. Architecture of calling ArcObjects of ArcGIS Server in Web Application

\subsection{Flex and BlazeDS}

Adobe Flex is a software development kit (SDK) released for developers to develop and deploy cross-platform RIA (Rich Internet Applications). Developers can use the MXML (an XML-based markup language) to lay out graphic user interfaces and use ActionScript to achieve interactivity. The codes would finally be compiled to Flash files which has got wide support and has affluent expressive force. By using the computing ability of client computer to solve multi-step processing, client-side validation, data management and display, the communication times between clients and server can be reduced and web application will become responsive [13-15].

BlazeDS is an open licensed java remoting and web messaging products of Adobe to simply developers' work to access back-end distributed data or push data to the RIA client. A BlazeDS application consists of two parts: a client-side application and 
a server-side J2EE web application. The client-side can be realized by Flex or Ajax libraries. Flex provide components such as HTTP Service, Web Services, Remote Object, Producer and Consumer to communicate with the BlazeDS server. The BlazeDS server running in J2EE Server container can provide proxy service, RPC service, messaging service and service adapter. Channels which encapsulate message formats, network protocols, and network behaviors are used by client to communicate with endpoints on sever. BlazeDS provides several types of channels, including standard and secure Action Message Format (AMF) channels and HTTP (AMFX) channels. AMF and HTTP channels support non-polling request-response patterns and client polling patterns to simulate real-time messaging [16-17].

\section{System Realization}

\subsection{Realization Scheme}

The MMSFEFW is of B/S (Browser/Server) structure and realized with J2EE technology. The client interface was built using Flex Builder and BlazeDS is employed to finish the communication between clients and server. The web module in the application logic layer was realized using Struts2 and the Axis2 was used to implement Web Service in this layer. ArcGIS Server 9.3 is adopted to act as the GIS Server to provide spatial services and spatial functions. ArcSDE and Oracle is used to manage the data.

\subsection{Realization of Geoprocessing}

ArGIS Server allow users to publish model or script tools created in ArcTool Box, or map document container tool layers to server to provide different kinds of geoprocessing service(GP Service). In addition more flexible geoprocessing can be realized by invoking the server APIs in program. In MMSFEFW both methods were used to process and analyze the sampling data.

Geoprocessing Using GP Service of ArcGIS. A GP service in ArcGIS Server is created in two steps. First, build geoprocessing tools on ArcGIS Desktop using model or script tools or creating a map with the built tools as tool layer. In these step much attention need to be paid to following three points: (1) the data types of input parameters and output parameters because unlike the desktop environment only some certain data types are supported on web application clients. (2) the place where middle data and the result data will be stored. (3) how the result will be displayed to the clients. The second step is to publish created tools or map with tool layer. The parameter defining the way in which the service will run and interact with the clients can be set in this step. The analysis of distribution of farmland pollution is realized in this method.

Fig 4 shows the model to using IDW as the spatial interpolation method to analyzing the spatial distribution of harmful substance. The flow of this model have three steps: firstly filter the samples data so that only the data to which the current user at least has read privilege will be used; secondly apply IDW method to the selected sample points within a certain spatial extent; Thirdly classify the results of IDW method according to the thematic classes set by users and set the color scheme of the output. 
The Inputs of the model include userids, Extent, Z_value, Cell_size, Reclass_Idw and symbol. Userids are numeric identifies of users whose sampling and plots data can be read by the current user, Extent represent the spatial extent to apply the spatial interpolation, the $\mathrm{z}_{-}$value represents the attribute sample points to be analyzed. The Cell_size assign the actual size each pixel represents. The Reclass_Idw and symbol respectively contains the classes division and symbology set with functions described in 2.2 section. The two outputs of this geoprocessing tool are named Idw_sp_650001 and Recess_Idw which are all of Raster type. Idw_sp_650001 is the result of IDW spatial interpolation method. Reclassfication is the result of reclassifying of Idw_sp_650001 according the input parameter Reclass_Idw.

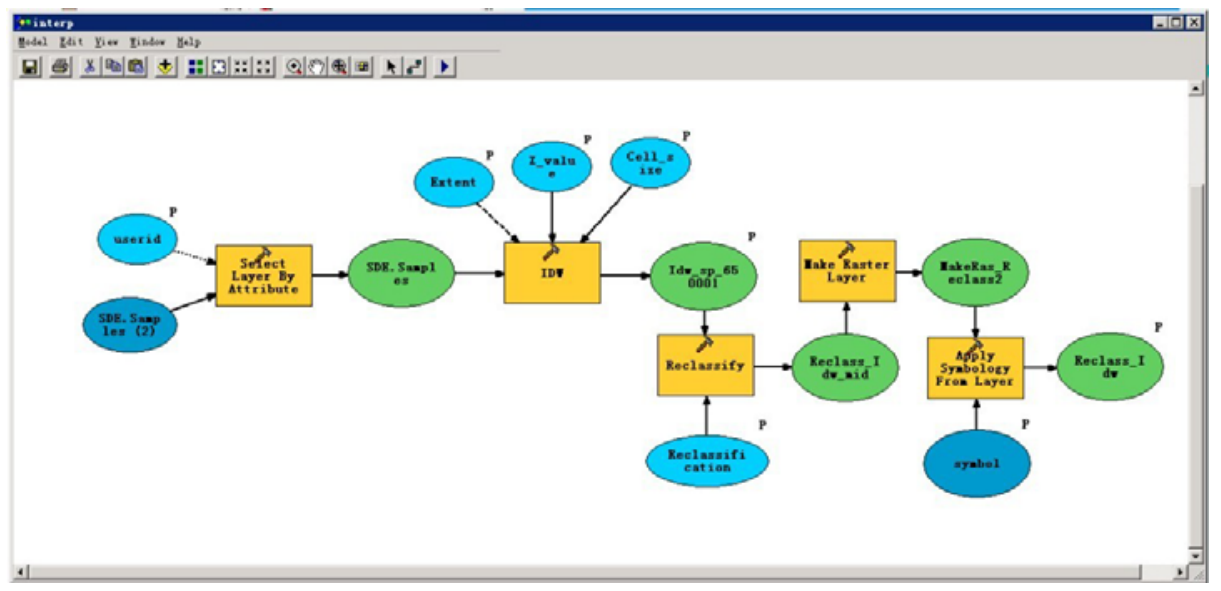

Fig. 4. Geoprocessing Tool

The accomplished model can then be published to the ArcGIS Server to provide geoprocessing service. The geoprocessing services can be directly called in flex. Following is the codes snippets calling the spatial interpolation service in asynchronous mode. The interpolation function calls the spatial interpolation service and the jobCompleteHandler function retrieve the result after the task completed. The following is the code snippet of clients to call the service.

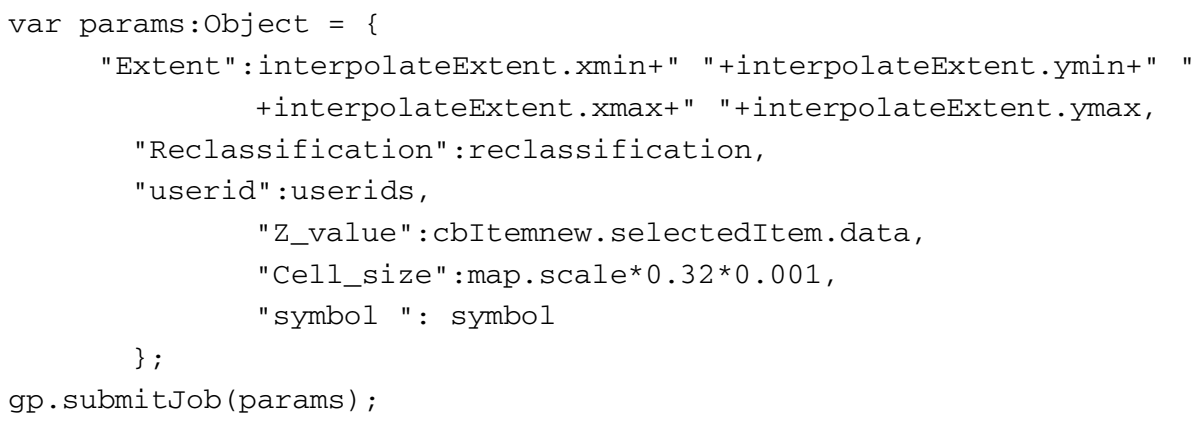


Geoprocessing Based on Server APIS. The quality evaluation of the farmland plots is realized by calling ArcObjects of ArcGIS Server. It's finished in the following 2 steps:(1) user selects the farmland plots to be evaluated using the select tool and the parameters describing the selection are sent to the Web Server, then the Web Server communicate with the ArcGIS Server to get the geometries of the selected plots and return the geometries fetched to the client to highlight the selected plots;(2) user fires the evaluate function through the client interface, and in answer to the evaluation request the Web Server first fetches samples points data in each selected plots from the ArcGIS Server, then uses the fetched data to evaluate the quality of each selected farmland plots by calling spatial analysis functions of the ArcObjects, and finally returns the evaluation to the Flex client to display to the user.

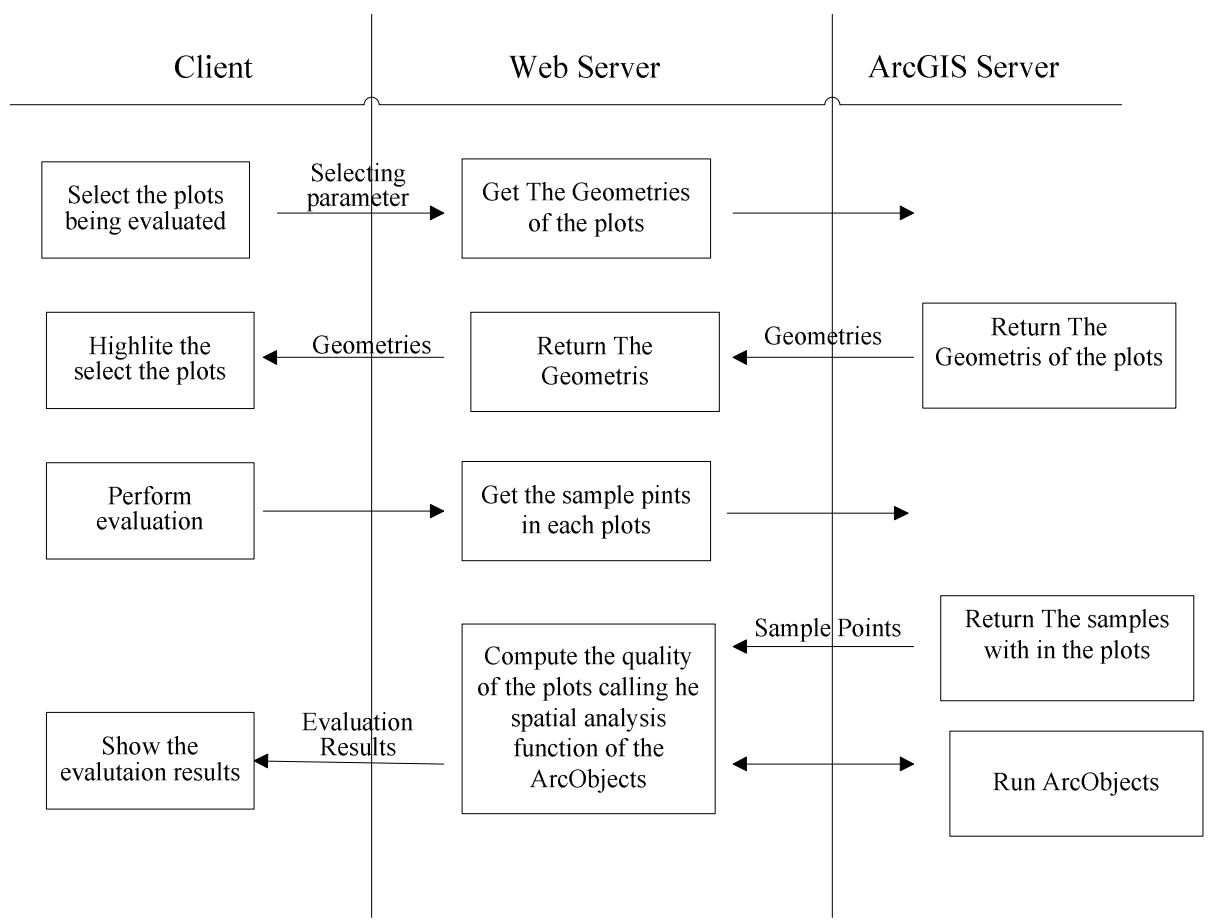

Fig. 5. The interaction among client, web application and ArcGIS Server during the operation evaluating the quality farmland plots

\subsection{User Structure and the Privileges Managements}

The tertiary user structure was designed realized MMSFEFW, shown in Fig 6. Users are grouped into different user groups, and each group has group administrators who are responsible for management of the users and data belonging to their group. The users and the user groups forms the lower two layers of the structure. At the top of the structure are the system administrators who have the highest controlling authority, responsible for configuration of the system and management of the user groups. 
In order to protect the rights of system users, strict privileges management scheme are adopted. Each user is assigned one or more roles which define the operations that can be performed and the data can read or written. A user with the reader role can only view, query or perform some spatial analysis on the environmental sampling data or farmland plots data belonging to users in the same group. He cannot input, update or remove the data. A user with the writer role can input environmental sampling data or farmland plots data, update, remove and group the data belonging to him besides what a user only with a reader role can perform. A user with the group administrator role can update, remove and group all the data belonging to the users of the group besides what user with a writer role can do. Users with the system administrator role have the highest controlling authority, that's to say they can use all functions the system provided and manage all the data the system have.

Every user in the system has the privilege to alter his own information except which group he belongs to. A group administrator can add, delete, and alter users in his group. The system administrator can add, delete and alter user groups and group administrators.

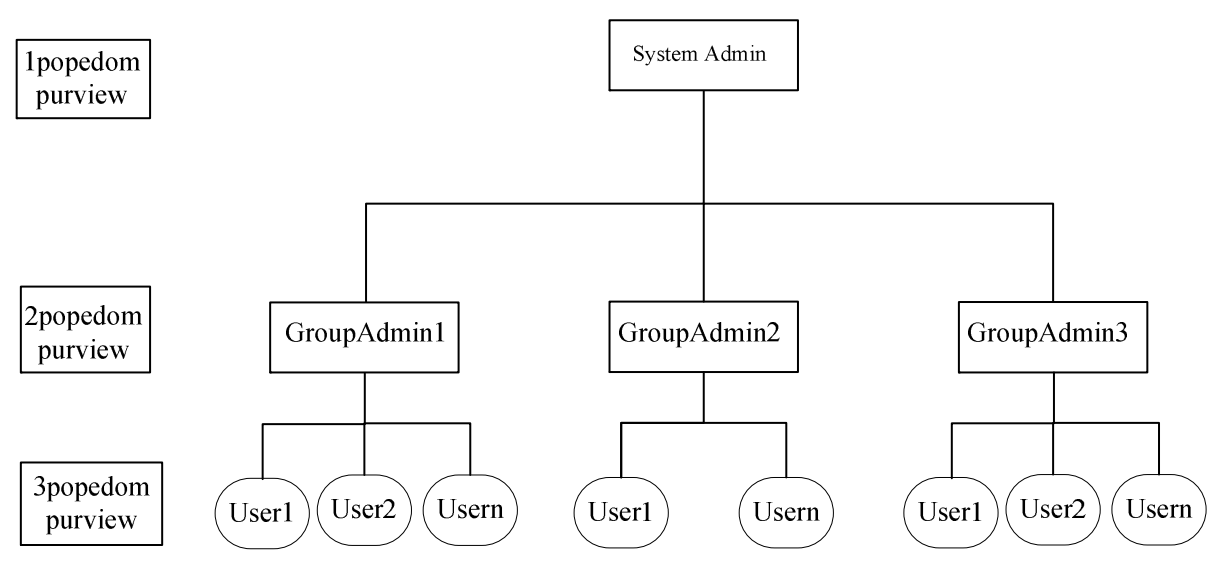

Fig. 6. Tertiary User Structure

\subsection{Results}

The interface of MMSFEFW uses the national map of China as background, and provide map control panel, menu bar, toolbox and information window. The menu contents differs after logging in according to the user's roles and privileges and the map will scale automatically to the extents of sampling data to which he has at least read privilege. Fig 7, Fig 8 and Fig 9 are the screenshots of MMSFEFW. Fig 7 shows the thematic map of $\mathrm{Cu}$ contents in each sampling points. Fig 8 shows the environmental quality evaluation results of the selected farmland plots. Fig 9 shows the spatial interpolation results of $\mathrm{Cu}$ contents in the specified area. 


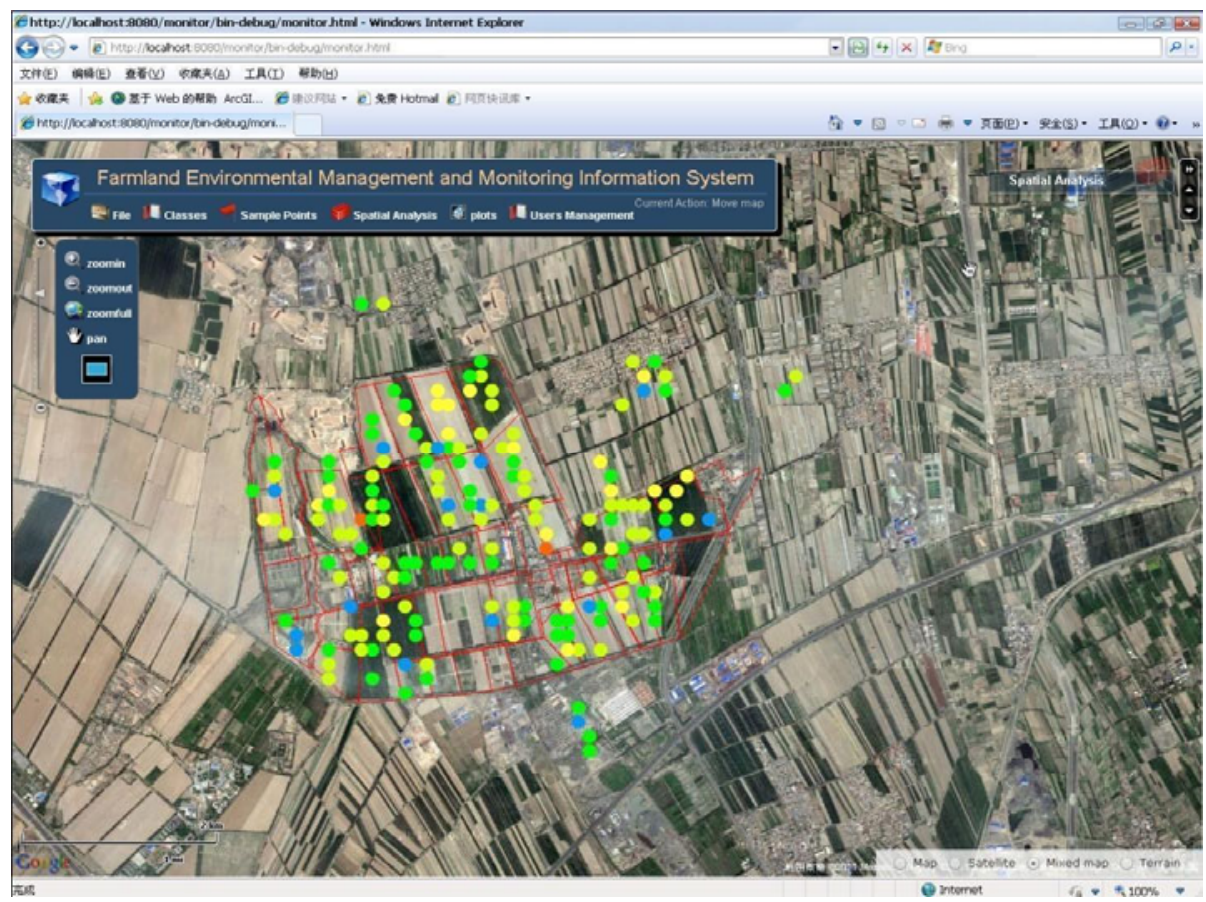

Fig. 7. Thematic map of copper contents in each sampling points

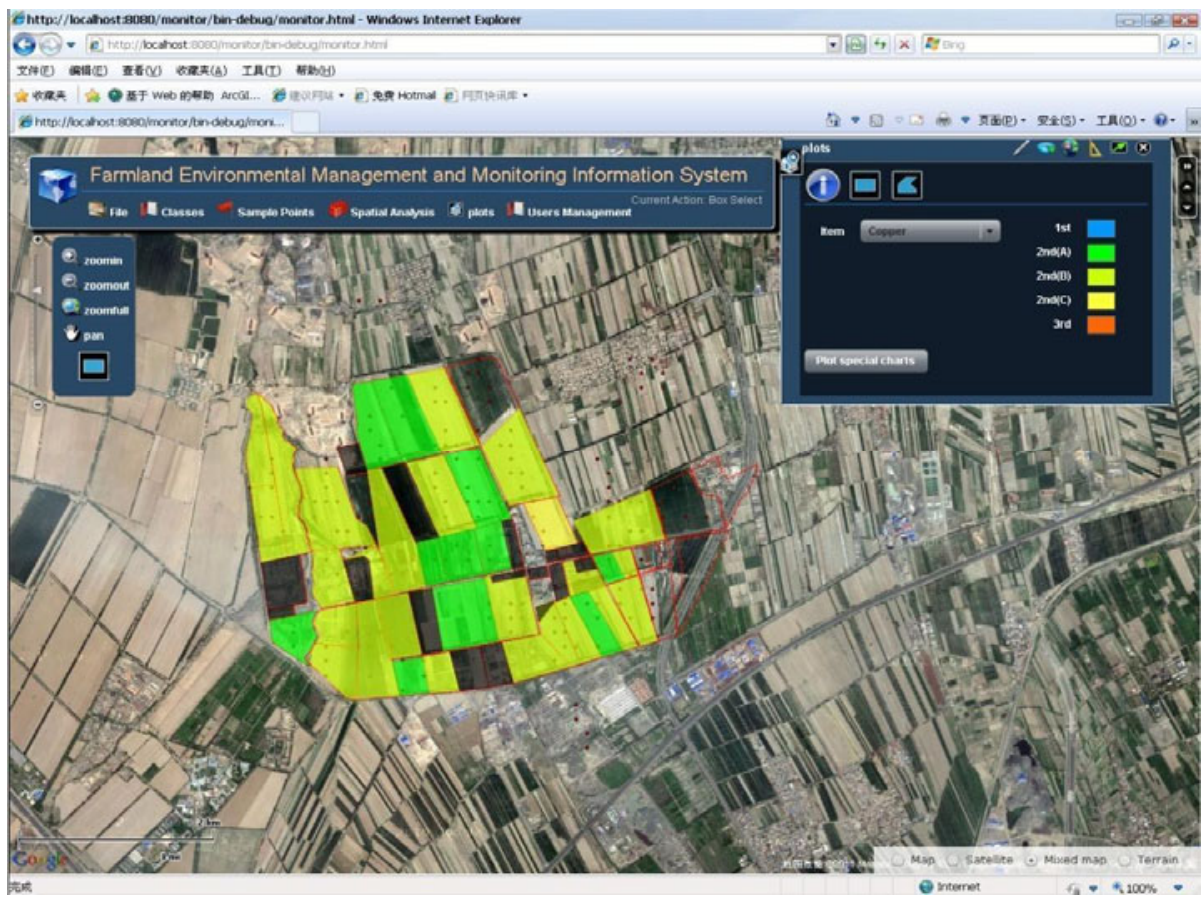

Fig. 8. Environmental quality evaluation results of the selected farmland plots 


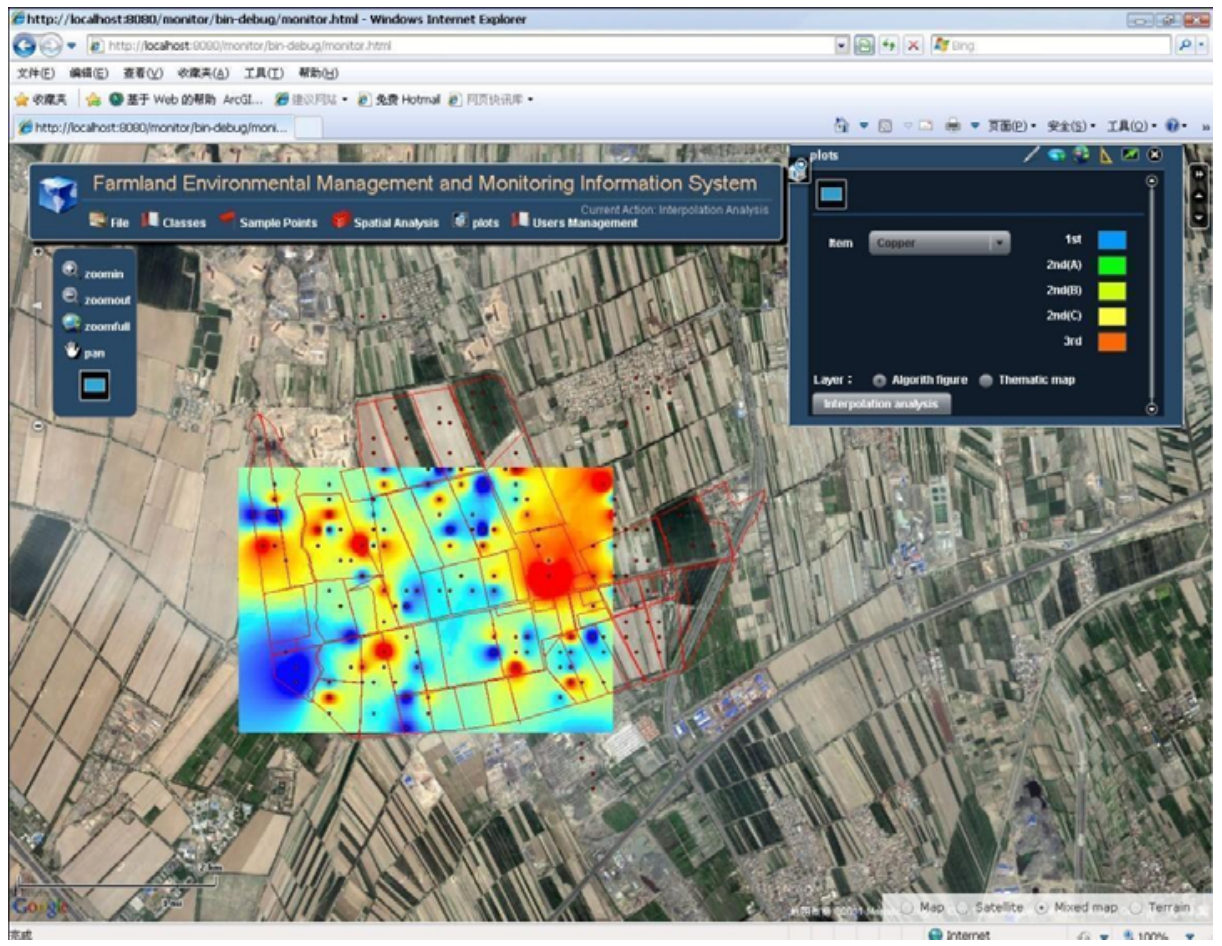

Fig. 9. Spatial interpolation results of $\mathrm{Cu}$ contents in the specified area

\section{Conclusions and Future Work}

In order to manage, visualize and analyze the sampling data of farmland environment, to mine the potential useful information from the collected data, and to guide the agricultural environmental management and decision making, the design and implementation of MMSFEFW and the key technologies used are introduced in this paper.

The MMSFEFW built using Flex, Web Services, ArcGIS Server and J2EE includes data layer, services layer and presentation layer. The flex was employed to realize the user interface. The spatial visualization and analysis were realized by directly calling configured rest services in flex clients or invoking ArcObjects of ArcGIS Server. To protect user's copyrights on sampling data, tertiary user structure was design and strict privileges management was implemented.

Further works include: 1) additional kinds of environmental data; 2) new environmental analysis models to provide better results for decision-making.

Acknowledgments. This research was supported by Beijing Municipal Natural Science Foundation (4102022) and key Projects for Constructing Scientific and Technological Innovation Ability of Beijing Academy of Agriculture and Forestry Sciences. The authors would also like to thank any reviewers for their constructive comments and suggestions. 


\section{References}

1. Shi, Z., Wang, F.: Effects of Pesticide Contamination on Microbial Diversity. Journal of Anhui Agricultural Sciences 35(19), 5840-5841, 591 (2007) (in Chinese)

2. Chen, Y.X., Lin, Q., He, Y.F., Tian, G.M.: Behavior of $\mathrm{Cu}$ and $\mathrm{Zn}$ under combined pollution of 2,4-dichlorophenol in the planted soil. Plant and Soil 261, 127-134 (2004)

3. Li, D., Wu, Z., Liang, C.: Soil Environmental Pollution and Agricultural Product Quality. Bulletin of Soil and Water Conservation 04 (2008) (in Chinese)

4. Wang, L., Gu, G., Zhang, M.: Present situation and problems in studies on environmental qualities of agricultural products producing sites in China. Anhui Agricultural Science Bulletin 12(12), 49-51 (2006) (in Chinese)

5. Huang, W., Cai, Q., Huang, M., et al.: Portable Unit for Rapid Determination of Pesticides Residues in Vegetables and Fruits. Journal of Instrumental Analysis 19(6), 87-89 (2000) (in Chinese)

6. Serpil, Y.K.: Validation and uncertainty assessment of rapid extraction and clean- up methods for the determination of 16 organochlorine pesticide residues in vegetables. Analytica Chemica Acta 571(5), 298 (2006)

7. Liang, T.G., Chen, Q.G., Ren, J.Z., et al.: A GIS-based expert system for pastoral agricultural development in Gansu province, PR China. New Zealand Journal of Agricultural Research 47(3), 313-325 (2004)

8. McCarthy, J.D., Graniero, P.A., Rozic, S.M.: An integrated gis-expert system framework for live hazard monitoring and detection. Sensors 8, 830-846 (2008)

9. ESRI China(Beijing).: The Best of REST Using REST in ArcGIS Server 9.3 (2009)

10. ESRI. Overveiw of ArcGIS for Server (EB/OL), http: / / www.esri.com/software/arcgis/arcgisserver/index.html

11. ESRI. ArcGIS API for Flex online Help (EB/OL), http: //resources.esri.com/arcgisserver/apis/flex

12. ESRI. The ArcGIs Server and image server architecture (January 2009)

13. Jiang, T.: Design and Realization of Enterprise Web Application System Based on Flex3. China Machine Press, Beijing (2008) (in Chinese)

14. Longley, P., Batty, M.: Advanced Spatial Analysis. ESRI Press, Redlands (2003)

15. Webster, S., McLeod, A.: Developing Rich Clients with Macromedia Flex. Peachp it Press (2004)

16. Cardell-Oliver, R., Smettem, K., Kranz, M., et al.: Field Testing a Wireless Sensor Network for Reactive Environmental Monitoring. In: Proceedings of the International Conference on Intelligent Sensors. Sensor Networks and Information Processing, pp. 7-12 (2004)

17. Fukatsu, T., Hirafuji, M.: Field Monitoring Using Sensor - Nodes with a Web Server. Journal of Robotics and Mechatronics 17(2), 164-172 (2005) 